\title{
Measurement quality and uncertainty evaluation in civil engineering research
}

\author{
A. Silva Ribeiro ${ }^{1, \star}$, J. Alves e Sousa ${ }^{2}$, C. Oliveira Costa ${ }^{1}$, J.F. Vieira de Sousa ${ }^{2}$, \\ J. Almeida Garrett ${ }^{1}$, and L. Lages Martins ${ }^{1}$ \\ 1 Laboratório Nacional de Engenharia Civil, Av. do Brasil 101, 1700-066 Lisboa, Portugal \\ 2 Laboratório Regional de Engenharia Civil, Rua Agostinho Pereira de Oliveira, 9000-264 Funchal, Portugal
}

Received: 12 April 2012 / Accepted: 16 July 2013

\begin{abstract}
Civil engineering is a branch of science that covers a broad range of areas where experimental procedures often plays an important role. The research in this field is usually supported by experimental structures able to test physical and mathematical models and to provide measurement results with acceptable accuracy. To assure measurement quality, a metrology probabilistic approach can provide valuable mathematical and computational tools especially suited to the study, evaluation and improvement of measurement processes in its different components (modeling, instrumentation performance, data processing, data validation and traceability), emphasizing measurement uncertainty evaluation as a tool to the analysis of results and to promote the quality and capacity associated with decision-making. This paper presents some of the research held by the metrology division of the Portuguese civil engineering research institutes, focused on the contribution of measurement uncertainty studies to a variety of frameworks, such as testing for metrological characterization and physical and mathematical modeling. Experimental data will be used to illustrate practical cases.
\end{abstract}

Keywords: Measurement uncertainty; quality; civil engineering

\section{Introduction}

Civil engineering is a branch of science that covers a broad spectrum of domains where the experimental component often plays an important role. Research in this field is usually supported by a strong experimental infrastructure whose quality depends, among other factors, on the accuracy of the measurements obtained using traceable instruments with high levels of accuracy.

Measurement can in this environment be a complex process involving different steps, all contributing to its accuracy, namely, the quality of physical and mathematical modeling, the performance of the instrumentation, the processing and validation of data, the traceability, the measurement uncertainty evaluation and the analysis and interpretation of measurands results.

Metrology can provide valuable mathematical and computational tools especially useful for the study, evaluation and improvement of measurement processes, promoting the quality and capacity associated with decision-making.

This paper aims to describe some of the research held by the metrology division of the Portuguese civil engineering research institutes, including some of the

\footnotetext{
${ }^{\star}$ Correspondence: asribeiro@lnec.pt
}

mathematical and computational tools used as well as some practical applications.

\section{Research topics}

Among the research areas in civil engineering this paper will focus on three research topics that, somehow, reflect the diversity of the lines of investigation in this field.

One of those areas is testing applied to geotechnical engineering, being the example under study taken from research related to triaxial compression tests in water saturated soils [1], where it will be necessary to make use of uncertainties from regression analysis [2], with the additional advantage of commonality with other applications.

The second topic concerns transportation and is based on a study developed to evaluate the thermal expansion of a pavement test specimen type, using strain gauges with specific thermal expansion coefficients. This example is related to materials testing in order to provide information regarding the influence of extreme environmental conditions such as temperature and humidity in processes of material aging, emphasizing the relevance of traceability and mathematical modeling to characterize and to review testing results.

The third example concerns the use of dimensional and geometrical contributions to the definition of concrete 
testing specimens with assured quality in order to support research studies. In this topic, the contribution is given by the characterization of geometrical quantities of metal and steel moulds [3,4] used to manufacture these specimens.

\section{Mathematical and computational tools}

Experimental data analysis is a key activity in most measurement processes. It involves developing a mathematical model of a physical system in terms of mathematical equations involving parameters that describe all relevant aspects of the system. The model specifies how the system is likely to respond to input data and the nature of the uncertainties associated with this data. This latter aspect is very important, and it is increasingly recommended to perform data analysis estimating the uncertainties associated with it $[4,5]$.

Regarding the geotechnical example, the study concerns the use of uncertainties obtained from a regression analysis, followed by an implicit scheme to propagate those uncertainties in a problem with two output quantities in a two-stage procedure. This type of problem has received adequate attention in a recent publication [6].

The quality of pavement materials is often related with its behaviour when subjected to mechanical testing, with its deformation sometimes measured using strain gauges mounted on the specimens. Considering that both elements are affected by temperature, the difference between thermal expansion coefficients should be considered as a contribution to the mathematical models adopted and, therefore, its metrological characterization required to take this contribution into account.

The requirement of materials aging testing includes the establishment of temperature and humidity conditions. It requires the instrument calibration in order to provide traceability that embodies the accuracy assurance within the SI units and, simultaneously, to provide reference values that should be used as information input variables to the mathematical models and for the study of spatial and temporal testing conditions.

The repeatability of testing is usually strongly related to the quality of test results. To achieve it the measurement process must be controlled from the start. In the case of testing the concrete properties, the testing procedure implies that the specimens should be provided with assured dimensional and geometrical tolerances to avoid bias results due to their own variability. In this case, the moulds used to manufacture the specimens should be measured and several geometrical quantities compared with standard tolerances, taking into account the measurement uncertainty as part of the conformity assessment.

Measurement performed in situ is usually strongly dependent on influencing conditions, namely, temperature, humidity and pressure. In the activity related to the monitoring of large structures, strain gauges are among the most widely used sensors. Its variation due to temperature effect is also significant, therefore, becomes particularly relevant to understand how temperature measurement uncertainty can affect, in some conditions, strain measurement uncertainties. This will help users to select sensors and to establish the required accuracy for the measurement systems.

\section{Metrology contribution to measurement quality in civil engineering research activities}

It is widely accepted today that metrology is one of the pilars that hold Quality. Measurement accuracy achieved, by this way, a relevant role in the Quality framework.

Recent developments towards a probabilistic interpretation of measurement, consolidated with the ISOGUM [1] publishing in 1993, allowed an increasing growth of studies related with how to properly express accuracy and to promote conformity assessment. The concept of measurement uncertainty was found as a major contribution to both needs.

The application of these concepts to the "real world" of different branches of Science, with all its complexity, created the need to try or develop many of the mathematical tools already available in order to find ways to solve the proposed problems.

Today, metrology can provide interesting solutions to deal with some of the high requirements of accuracy found on civil engineering research. A few examples on its contribution to the overall Quality will be detailed in this section.

\subsection{Geotechnical testing case study}

In this particular study attention will be paid to triaxial compression tests used to typify particular soils. These tests involve having the soil specimen loaded to failure, by compression, when submitted to a specified confined stress. Through a series of tests, usually three (scarcity of data is a relevant point), with different confinement stresses, the required experimental data to draw the failure line (stress coordinates representing failure, see Fig. 1) are obtained, from which it is possible to determine its intercept and the slope. This information is then used to determine two parameters that characterize the soil, namely, the cohesion and the friction angle.

For the present study isotropic consolidated and drained triaxial compression tests were performed on specimens that had been previously saturated, according to the CEN ISO/TEC 17892-9 [1] standard. The specimens were obtained by compaction, for the same moisture content and compaction energy, so that the same bulk density was obtained. The specimens were compacted with a diameter close to $70 \mathrm{~mm}$, and a height/diameter relation close to 2 (the test standard recommends this relation to be in the interval $[1.85,2.25])$.

In the context of this study nine specimens were tested, with consolidation stress values of $100 \mathrm{kPa}, 200 \mathrm{kPa}$ and $300 \mathrm{kPa}$, to allow a comparison of the common situation of having three specimens, one at each consolidation stress 


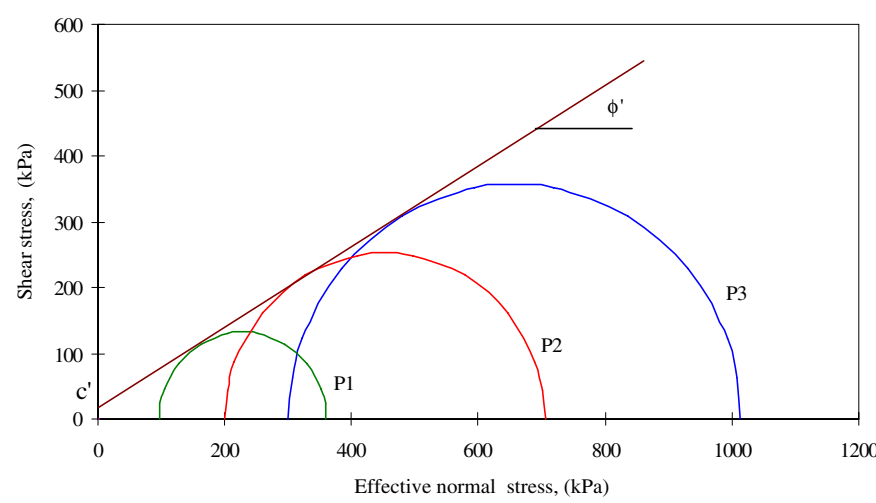

Fig. 1. Evaluation of the resistance parameters using Mohr circles at failure.

Table 1. Results of tests for different consolidation stresses.

\begin{tabular}{cccc}
\hline Specimen & P1 & P2 & P3 \\
\hline Consolidation stress $/ \mathrm{kPa}$ & 100 & 200 & 300 \\
$q^{\prime}$ at failure $/ \mathrm{kPa}$ & 132 & 252 & 355 \\
$p^{\prime}$ at failure $/ \mathrm{kPa}$ & 228 & 452 & 655 \\
\hline
\end{tabular}

value (baseline condition), with a situation where there is a double repetition at each of those testing points. In this work only the results of the shear phase will be presented, in which, among others, the quantities measured include: horizontal (radial) stress $\left(\sigma_{3}\right)$, pore pressure $(u)$, axial force $(P)$, height variation $(\Delta H)$ and volume variation $(\Delta V)$.

In practice, it is common to represent the failure stress condition just by a single point, in a $(p, q)$ or $\left(p^{\prime}, q^{\prime}\right)$ coordinate system, where

$$
\begin{aligned}
& p=\left(\sigma_{1}+\sigma_{3}\right) / 2, \quad p^{\prime}=\left(\sigma_{1}^{\prime}+\sigma_{3}^{\prime}\right) / 2, \\
& q=q^{\prime}=\left(\sigma_{1}-\sigma_{3}\right) / 2=\left(\sigma_{1}^{\prime}-\sigma_{3}^{\prime}\right) / 2 .
\end{aligned}
$$

In Table 1 we display the consolidation stresses and also the failure values of $p^{\prime}$ and $q^{\prime}$ for the same tests. Figure 3 displays the more common graphical representation of a straight-line regression, obtained by ordinary least squares, whose parameters (slope and intercept) are the basis for the evaluation of the friction angle $\phi^{\prime}$ and the cohesion $c^{\prime}$ [1], obtained using an Excel spreadsheet.

The usual approach is to determine the resistance parameter values, from such a straight-line regression by an ordinary least squares approach, without uncertainty associated with them. In this case, the data displayed in Figure 2 yields $\phi^{\prime}=\arcsin (\tan \alpha)=31.5$ and $c^{\prime}=$ $a / \cos \left(\phi^{\prime}\right)=16.2$.

In the case of the triaxial compression test, the output testing results - friction angle and cohesion - are based, as mentioned earlier, on the slope of the straight-line regression function and its intercept with the ordinate axis. This function is in general established based on just three points, as is normal practice in this type of test. The ordinate and abscissa axes represent the shear stress and the effective mean normal stress, respectively. These quantities, on the other hand, are calculated using expression (1)

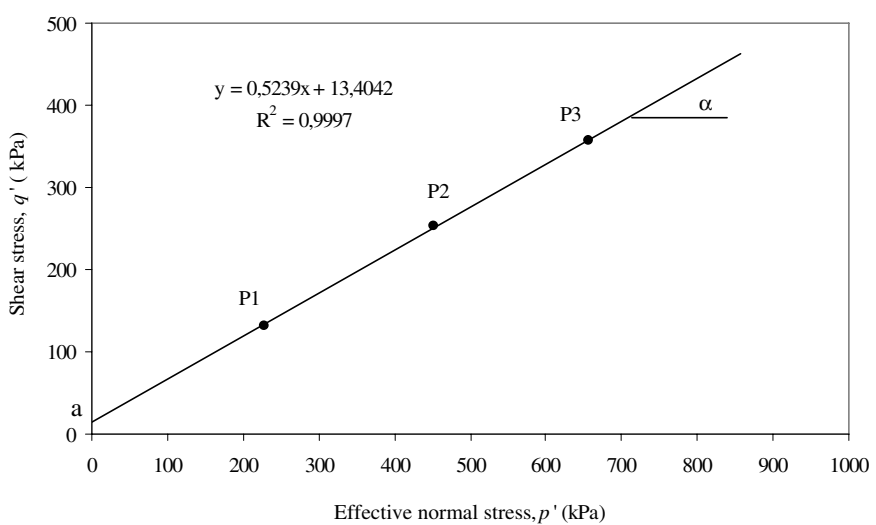

Fig. 2. Straight-line regression for the evaluation of the friction angle $\phi^{\prime}$ and the cohesion $c^{\prime}$.

Table 2. Results from MCM.

\begin{tabular}{ccccc}
\hline Specimen & $q^{\prime} / \mathrm{kPa}$ & $u_{q^{\prime}} / \mathrm{kPa}$ & $p^{\prime} / \mathrm{kPa}$ & $u_{p^{\prime}} / \mathrm{kPa}$ \\
\hline 1 & 132 & 1.63 & 228 & 0.47 \\
2 & 252 & 1.63 & 452 & 0.47 \\
3 & 355 & 1.62 & 655 & 0.47 \\
\hline
\end{tabular}

and the following expressions:

$$
\begin{aligned}
\sigma_{1}^{\prime} & =\sigma_{1}-u, \quad \sigma_{3}^{\prime}=\sigma_{3}-u, \\
\sigma_{1} & =\sigma_{3}+\frac{P}{A} .
\end{aligned}
$$

The pore pressure $u$ in the specimen, indicated by expression (2), relates the total stress and the effective stress, not only for the axial stress $\left(\sigma_{1}\right)$ but also for the horizontal stress $\left(\sigma_{3}\right)$. Substituting in (1) the terms of the axial and horizontal stresses by their definitions (2) and (3), the following expressions (4) and (5) are obtained, with which the uncertainty evaluation will be performed:

$$
\begin{aligned}
q^{\prime} & =\frac{P}{2 A}, \\
p^{\prime} & =\frac{2 \sigma_{3}-2 u+(P / A)}{2},
\end{aligned}
$$

where the area $A$ is calculated using expression (6):

$$
A=\frac{V_{i}-\Delta V_{C}-\Delta V}{H_{i}-\Delta H_{C}-\Delta H} .
$$

It should be noted that the evaluation of the area $A$ of the specimen involves quadratic terms ([1], p. 18), which brings a degree of complexity to the evaluation of the sensitivity coefficients needed for applying the GUM uncertainty framework. The assumption of linearity, in this case, is no longer valid.

The point pairs $\left(p^{\prime}, q^{\prime}\right)$ and the respective uncertainties of Table 2 are the input data for the application of the least squares method to a straight-line regression for the 3 points considered [2].

The application of this methodology to the data of Table 2 , that is to the pairs of points $\left(p^{\prime}, q^{\prime}\right)$ and respective 
Table 3. Results of straight-line regression for specimens 1 to 3 .

\begin{tabular}{ccccc}
\hline & $\phi^{\prime} /{ }^{\circ}$ & $u_{\phi^{\prime}} /^{\circ}$ & $c^{\prime} / \mathrm{kPa}$ & $u_{c^{\prime}} / \mathrm{kPa}$ \\
\hline No validation & 31.6 & - & 16.7 & - \\
\hline After validation & 31.6 & 0.63 & 16.7 & 5.13 \\
\hline
\end{tabular}

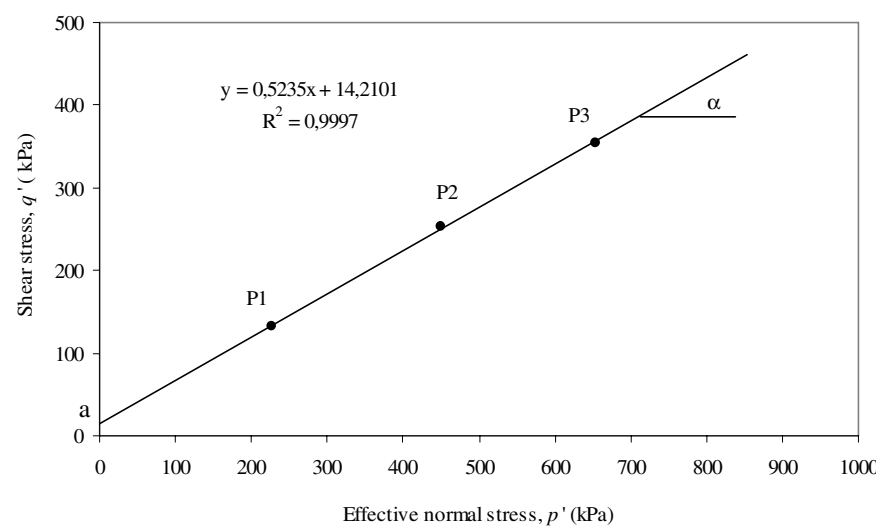

Fig. 3. Straight-line regression with uncertainties evaluated using MCM.

Table 4. Results of straight-line regression for specimens 1 to 5 with experimental dispersion.

\begin{tabular}{ccccc}
\hline & $\phi^{\prime} /{ }^{\circ}$ & $u_{\phi^{\prime}} /{ }^{\circ}$ & $c^{\prime} / \mathrm{kPa}$ & $u_{c^{\prime}} / \mathrm{kPa}$ \\
\hline Validation accepted & 30.6 & 0.36 & 22.12 & 2.55 \\
\hline
\end{tabular}

uncertainties, produced the values of $a, b, \phi^{\prime}$ (friction angle) and $c^{\prime}$ (cohesion) shown in Table 3 and in Figure 3.

The validation referred to in the previous table is established by comparing the observed $R$ value with the chi-squared distribution with $v=m-2$ degrees of freedom and probability $95 \%$. As the observed $R$ value was outside the corresponding interval, a value $\hat{\sigma}^{2}=R /(m-2)$ was determined to adjust the matrix $U_{a}$ proportionally to this factor. The uncertainty values $u(a)$ and $u(b)$ were inflated in this way whereas the values of $a$ and $b$ remained unchanged.

Having available the experimental dispersion values related to the consolidation stresses of $100 \mathrm{kPa}, 200 \mathrm{kPa}$ and $300 \mathrm{kPa}$, for a total of 3 tests at each of these stresses, it was decided to use these values in two ways. First, the linear regression was repeated with these experimental uncertainties, much larger than the previous uncertainties, and the results in Table 4 and Figure 4 were obtained. Then, the friction angles and cohesion for each of these 3 tests were calculated and an estimative for the experimental dispersion of $\phi^{\prime}$ e de $c^{\prime}$ was obtained.

Note how the introduction of larger uncertainties in the model of linear regression led to the acceptance of the model validation, with no need for an adjustment, and how the uncertainties of $\phi^{\prime}$ and $c^{\prime}$, without the adjustment factor, resulted in lower values. The values of direct experimental standard deviation for $\phi^{\prime}$ and $c^{\prime}$ were of 0.92 and 5.9, respectively.

It is worth pointing out that the uncertainties associated with $\phi^{\prime}$ e de $c^{\prime}$ can be readily obtained if an

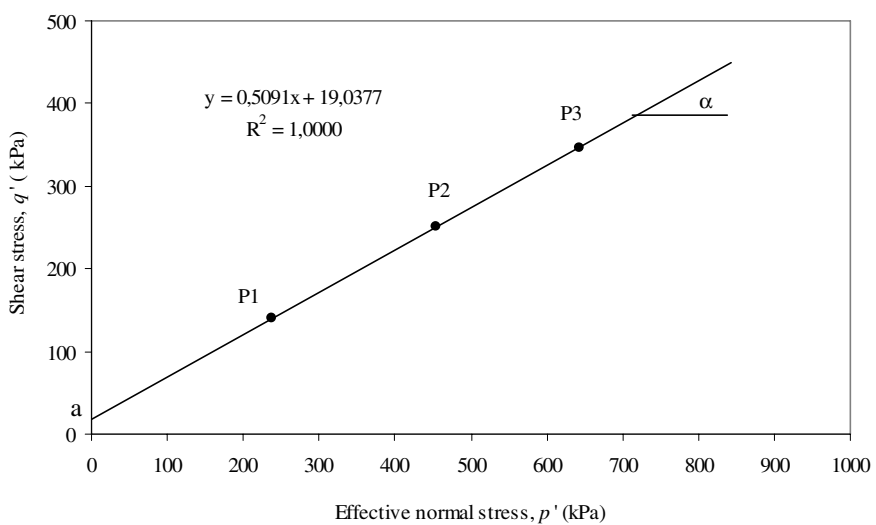

Fig. 4. Straight-line regression with uncertainties by experimental dispersion.

implicit scheme is implemented over $\phi^{\prime}=\arcsin (b)$ and $c^{\prime}=a / \cos \left(\phi^{\prime}\right)$ yielding two Jacobian matrices with sensitivity coefficients with respect to $\left(c^{\prime}, \phi^{\prime}\right)$ and $(a, b)$ :

$$
\begin{aligned}
& J_{y}=\left[\begin{array}{cc}
\cos (\phi) & -c \sin (\phi) \\
0 & \cos (\phi)
\end{array}\right] \\
& J_{x}=\left[\begin{array}{cc}
-1 & 0 \\
0 & -1
\end{array}\right]
\end{aligned}
$$

which in conjunction with the covariance matrix for $(a, b)$

$$
V_{x}=\left[\begin{array}{cc}
u^{2}(a) & u(a, b) \\
u(a, b) & u^{2}(b)
\end{array}\right]
$$

permits the propagation of uncertainty by solving $J y$. $V y \cdot J y^{T}=J x \cdot V x \cdot J x^{T}$ and extracting the standard uncertainty and covariance.

$$
\begin{aligned}
u(c) & =\left[V_{y}(1,1)\right]^{1 / 2} \\
u(\phi) & =\left[V_{y}(2,2)\right]^{1 / 2} \\
u(c, \phi) & =V_{y}(1,2) .
\end{aligned}
$$

The linear regression with uncertainties based on experimental dispersion results in a valid linear regression model, but ideally more replicates should be used. The uncertainties associated with the friction angle and the cohesion, when obtained solely by the experimental data are smaller than those obtained by generalised distance regression, with inflated (scaled) covariance matrix.

\subsection{Pavement materials specimen testing}

The study of pavement materials performance under extreme environmental conditions (of temperature and humidity) can be studied by testing pavement specimens to high and low temperature and humidity levels, using chambers specially suited to establish environments with thermal and humidity highly controlled conditions.

In order to obtain the behaviour of these specimens displacement sensors are required, being common the use 


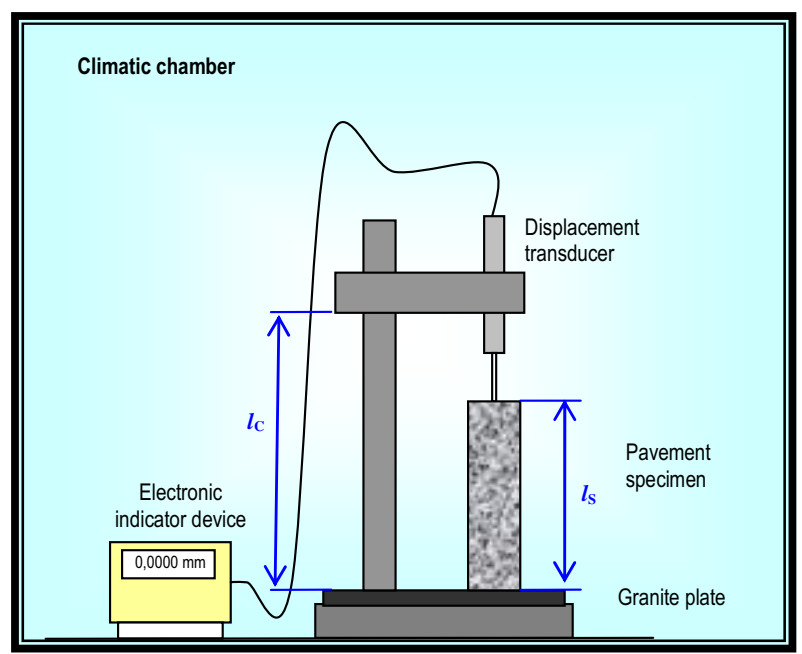

Fig. 5. Experimental setup for the evaluation of thermal expansion coefficient of pavement specimen.

of strain gauges connected to bridges to measure the displacement variations observed at the specimen faces.

The quality of the measurement is strongly related with the fact that both specimens and strain gauges have similar coefficients of thermal expansion, being these usually known with some accuracy for the strain gauges but not well known for the specimens worsened by the fact that the pavement material can be quite heterogeneous.

In order to obtain estimates of the specimens coefficient of thermal expansion $\left(\alpha_{s}\right)$, LNEC metrology division provided a setup to estimate that parameter for a set of pavement specimens. The setup had a $300 \mathrm{~mm}$ vertical column with horizontal arm and a granite base plate, a displacement transducer $(50 \mathrm{~mm}$ range and $0.1 \mu \mathrm{m}$ of resolution) with a digital device indicator and a chamber able to control temperature (stability and uniformity) within $0.1{ }^{\circ} \mathrm{C}$ and humidity within $0.5 \%$ rh.

The procedure developed was two stage, being the first one the evaluation of the setup column coefficient of thermal expansion $\left(\alpha_{c}\right)$, and the second one, the evaluation of the specimens coefficient of thermal expansion $\left(\alpha_{s}\right)$.

For the first step, three $100 \mathrm{~mm}$ pure metallic bars (invar, steel and aluminium) were used as reference materials with known $\alpha_{s}$ and the testing was carried doing the increment of temperature from $5{ }^{\circ} \mathrm{C}$ to $30{ }^{\circ} \mathrm{C}$ with $5^{\circ} \mathrm{C}$ steps.

Considering a change in temperature of $\Delta \theta$, the displacement $d$ measured by the transducer, for each temperature step and reference material, is approx. given by (7) and the estimates of the setup column $\alpha_{c}$ by (8).

$$
\begin{aligned}
d_{i} & =\left(l_{s} \alpha_{s} \Delta \theta_{i}\right)-\left(l_{c} \alpha_{c} \Delta \theta_{i}\right) \\
\alpha_{c} & =\frac{\left[\left(l_{s} \alpha_{s} \Delta \theta_{i}\right)-d_{i}\right]}{\left(l_{c} \Delta \theta_{i}\right)} .
\end{aligned}
$$

Calibration results are given in Table 5, being the measurement uncertainty obtained using first order GUM approach. The estimate obtained is according with the
Table 5. Experimental estimates of the thermal expansion coefficient for the setup.

\begin{tabular}{lcc}
\hline & $\alpha_{c} /{ }^{\circ} \mathrm{C}^{-1}$ & $u\left(\alpha_{c}\right) /{ }^{\circ} \mathrm{C}^{-1}$ \\
\hline $\mathrm{RM}$ (invar) & $1.16 \times 10^{-5}$ & $9 \times 10^{-7}$ \\
$\mathrm{RM}$ (steel) & $1.17 \times 10^{-5}$ & $8 \times 10^{-7}$ \\
$\mathrm{RM}$ (Al) & $1.18 \times 10^{-5}$ & $10 \times 10^{-7}$ \\
\hline
\end{tabular}

predicted value, considering that the main setup material is of stainless steel type.

The second stage was to perform the same testing using a sample of five specimens $(100 \mathrm{~mm}$ high and $20 \mathrm{~mm} \times 20 \mathrm{~mm}$ base square) from the same population to be tested, being their $\left(\alpha_{s}\right)$ obtained after correction of the setup column and transducer effects. The mathematical model is also obtained from (1), for the required unknown variable.

$$
\alpha_{s}=\frac{\left[\left(l_{c} \alpha_{c} \Delta \theta_{i}\right)+d_{i}\right]}{\left(l_{s} \Delta \theta_{i}\right)} .
$$

The analysis of the experimental results shown in Figure 6 may point out some conclusions:

i. The nonlinearity found avoids the need to consider a single estimator of the thermal expansion coefficient to be given to the specimens.

ii. Heterogeneity of material gives similar behaviour but different estimates for each temperature interval. It explains the memory effect observed on the decreasing cycle relative to the high temperature result and also the gap between the first and last points, both obtained at the same reference temperature.

iii. A reversibility effect was detected, with the displacements higher during the increase of temperature (top curves) than during the decrease of temperature.

iv. The analysis of temperature cycles appear to indicate that a drift exists, with the shift calculated as the difference between the final and the initial displacement of each cycle.

Regarding the measurement uncertainty, estimates were obtained again using first order GUM approach applied to equation (9), using as estimate the average of the setup column thermal coefficient given on Table $6\left(u\left(\alpha_{c}\right)=\right.$ $\left.9 \times 10^{-7}{ }^{\circ} \mathrm{C}^{-1}\right)$. The result obtained, expressed in Figure 6 , was of $\left(u\left(\alpha_{s}\right)=2.0 \times 10^{-6}{ }^{\circ} \mathrm{C}^{-1}\right)$.

Taking into consideration the studies carried out, it was possible to conclude that the use of extensometers to measure displacements in specimens used on road pavement testing, was within the measurement range of extensometers and their own thermal expansion coefficient. However, an accurate measurement of temperature during testing is strongly advisable.

In this process, the thermal coefficient of the glue (for several compositions) used to stick the extensometers to the specimens was also evaluated previously at laboratory, being obtained estimates between $1 \times 10^{-5}{ }^{\circ} \mathrm{C}^{-1}$ and $2 \times 10^{-5}{ }^{\circ} \mathrm{C}^{-1}$. Further studies are intended to be made in order to evaluate if the overall accuracy obtained is acceptable to measure the phenomena to be studied. 


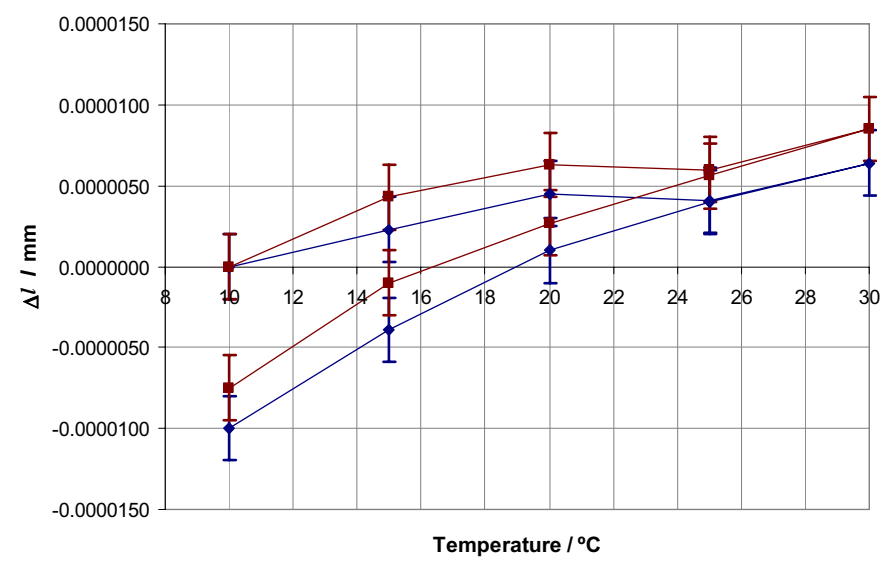

Fig. 6. Results of relative displacement measured for two tested specimens.

\subsection{Concrete moulds geometrical conformity assessment case study}

Concrete is a material widely used in many structures having specific requirements that imply physical and chemical testing. Due to its inhomogeneity, the quality of the testing depends on several conditions related to the process inherent to the cast of concrete testing specimens.

In this process one of the conditions that are required to assure reproducibility of the testing is the conformity assessment of the dimensional and geometrical quantities of moulds, considering that it can directly affect the quality of the specimens performance in testing.

Moulds can have different shapes (e.g., cubic, cylinder, prismatic and triple-gang) according to the applications and the international standards used $[3,4]$. In the studied case, the triple gang moulds, conformity requirements are found on EN 196-1 [4].

In the case of triple-gang moulds (see Fig. 7) each prismatic compartment should be in conformity with dimensional, flatness, squareness and roughness tolerances. The distance between joints at each compartment should be within $(40.0 \pm 0.2) \mathrm{mm}$, the length $(160 \pm 1) \mathrm{mm}$, the height $(40.1 \pm 0.1) \mathrm{mm}$, the flatness of each surface should be within $\pm 0.03 \mathrm{~mm}$, the squareness between each internal face and adjacent face or with bottom plane should be within $\pm 0.2 \mathrm{~mm}$ and the roughness should be higher than N8 class.

The metrological testing performed at LNEC can be applied to metal/steel moulds with cubic, prismatic, cylinder or triple-gang prismatic configuration, being supported on the use of a CMM 3D reference standard able to measure about 200 points along the mould planes within an expanded uncertainty of about $2 \mu \mathrm{m}$, providing the information needed to evaluate the required measurands and use the results to perform the conformity assessment.

The procedure developed at LNEC is supported on programmed routines that include four main actions:

- the initial setup of a reference axis origin for the 3D coordinate system;
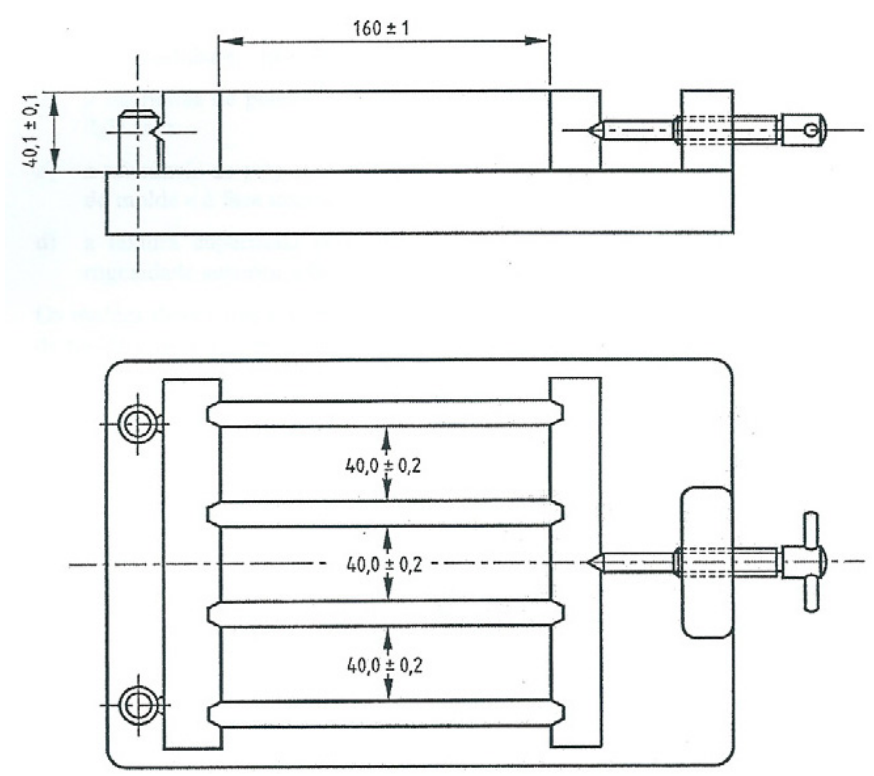

Fig. 7. Ttriple gang mold (EN 196-1 dimensions and tolerances in $\mathrm{mm}$ ).

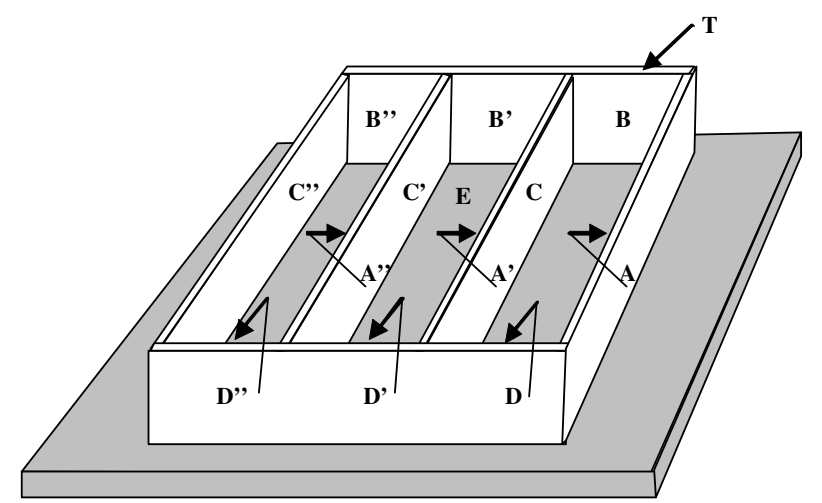

Fig. 8. Ttriple gang mold planes identification.

- the following sequence of displacements needed to measure several coordinates along the top plan $(\mathrm{T})$, the inside vertical planes (A, B, C, D) and the base plan (E) as described in Figure 8;

- the use of these coordinates to calculate the required dimensional and geometrical quantities;

- the conformity assessment.

A set of experimental results obtained for one compartment of a triple gang mould tested at LNEC are given in the following table.

Uncertainty plays a relevant role in the conformity assessment (CA) analysis, as it defines an interval comparable with the EN 196-1 standard tolerance interval, its magnitude should be small enough to assure the CA result.

The experimental procedure provides two types of results, the angle and the deviation. The latter is required to the conformity assessment process, which needs the values of estimates (see Tab. 6) and associated uncertainties (to be evaluated). 
Table 6. Experimental results obtained for a triple mold tested at LNEC's metrology laboratory.

\begin{tabular}{|c|c|c|c|}
\hline Measure & $\begin{array}{c}\text { Nominal } \\
\text { length and } \\
\text { tolerance }\end{array}$ & $\begin{array}{c}\text { Min. } \\
\text { experimental } \\
\text { value } / \mathrm{mm}\end{array}$ & $\begin{array}{c}\text { Max. } \\
\text { experimental } \\
\text { value } / \mathrm{mm}\end{array}$ \\
\hline $\mathrm{AC}$ & $(40.0 \pm 0.2) \mathrm{mm}$ & 39.956 & 39.973 \\
\hline $\mathrm{BD}$ & $(160 \pm 1) \mathrm{mm}$ & 160.051 & 160.011 \\
\hline $\mathrm{ET}$ & $(40.1 \pm 0.1) \mathrm{mm}$ & 40.167 & 40.142 \\
\hline Measure & $\begin{array}{l}\text { Nominal } \\
\text { angle and } \\
\text { tolerance }\end{array}$ & $\begin{array}{c}\text { Experimental } \\
\text { meas. } \\
\text { angle/으 }\end{array}$ & $\begin{array}{c}\text { Deviation/ } \\
\mathrm{mm}\end{array}$ \\
\hline$\angle \mathrm{AB}$ & 90 으 $0.2 \mathrm{~mm}$ & 89.987 & -0.009 \\
\hline$\angle \mathrm{BC}$ & $90 \stackrel{\circ}{-} 0.2 \mathrm{~mm}$ & 89.986 & -0.010 \\
\hline$\angle \mathrm{CD}$ & $90 \stackrel{-}{\circ} 0.2 \mathrm{~mm}$ & 90.001 & 0.001 \\
\hline$\angle \mathrm{DA}$ & $90 \stackrel{\circ}{\circ} / 0.2 \mathrm{~mm}$ & 90.002 & 0.015 \\
\hline$\angle \mathrm{AE}$ & 90 ㅇ $/ 0.2 \mathrm{~mm}$ & 89.994 & -0.004 \\
\hline$\angle \mathrm{BE}$ & $90 \stackrel{\circ}{\circ} 0.2 \mathrm{~mm}$ & 90.022 & 0.015 \\
\hline$\angle \mathrm{CE}$ & $90 \stackrel{\circ}{-} 0.2 \mathrm{~mm}$ & 89.992 & -0.006 \\
\hline$\angle \mathrm{DE}$ & $90 \stackrel{\circ}{\circ} 0.2 \mathrm{~mm}$ & 89.915 & -0.059 \\
\hline / / CA & $0 \stackrel{\circ}{-} 0.2 \mathrm{~mm}$ & 0.016 & 0.011 \\
\hline / / BD & $0 \stackrel{\circ}{0} 0.2 \mathrm{~mm}$ & 0.039 & 0.027 \\
\hline / / ET & $0 \stackrel{\circ}{\circ} 0.2 \mathrm{~mm}$ & 0.025 & 0.017 \\
\hline $\begin{array}{l}\text { Measure } \\
\text { (flatness) }\end{array}$ & $\begin{array}{l}\text { Nominal length } \\
\text { and tolerance }\end{array}$ & Deviation $/ \mathrm{mm}$ & \\
\hline Plan A & $\pm 0.03 \mathrm{~mm}$ & $\overline{0.009}$ & \\
\hline Plan B & $\pm 0.03 \mathrm{~mm}$ & 0.006 & \\
\hline Plan C & $\pm 0.03 \mathrm{~mm}$ & 0.008 & \\
\hline Plan D & $\pm 0.03 \mathrm{~mm}$ & 0.004 & \\
\hline Plan E & $\pm 0.03 \mathrm{~mm}$ & 0.010 & \\
\hline
\end{tabular}

In the first step of the procedure the reference CMM provides an angle, $\theta$, between every pair of planes, $P_{1}$ and $P_{2}$, constructed based on two sets of points, followed by the numerical evaluation of their normal vectors, $n_{1}$ and $n_{2}$, being:

$$
\cos \theta=\frac{\left|n_{1} \cdot n_{2}\right|}{\left\|n_{1}\right\|\left\|n_{2}\right\|} .
$$

The second step of the procedure is to use the estimate of each measured angle and of the length related to the maximum angular deviation, $l$, to evaluate the deviation related to orthogonality $\left(d_{\perp}\right)$ and to parallelism $\left(d_{\|}\right)$, respectively given by:

$$
\begin{aligned}
d_{\perp} & =l \tan \left(\frac{\pi}{2}-\theta\right) \\
d_{/ /} & =l \tan (\theta) .
\end{aligned}
$$

Considering the nonlinearity of the mathematical model, the use of Monte Carlo Method (MCM) was found suitable for the purpose of uncertainty evaluation. Simulations were performed for each of the experimental values presented in Table 6, using MatLab validated routines being performed numerical simulations with sequences of $10^{6}$ draws.

In the case of the measurement of the angle $\angle \mathrm{AB}$, the model applied was (11a), being the input variables introduced considering the length of $(40 \pm 1) \mathrm{mm}$, and a measured average angle of $(89.987 \pm 0.017)^{\circ}$, being the angle uncertainty obtained combining the CMM angular
Table 7. MCM results for $\angle A B$ orthogonality deviation.

\begin{tabular}{cc}
\hline & $\mathrm{mm}$ \\
\hline$d$ & $-9,1 \times 10^{-3}$ \\
$U_{95 \%}(d)$ & $0.6 \times 10^{-3}$ \\
Skewness $(d)$ & $<3 \times 10^{-2}$ \\
Kurtosis $(d)$ & $<2 \times 10^{-2}$ \\
\hline
\end{tabular}

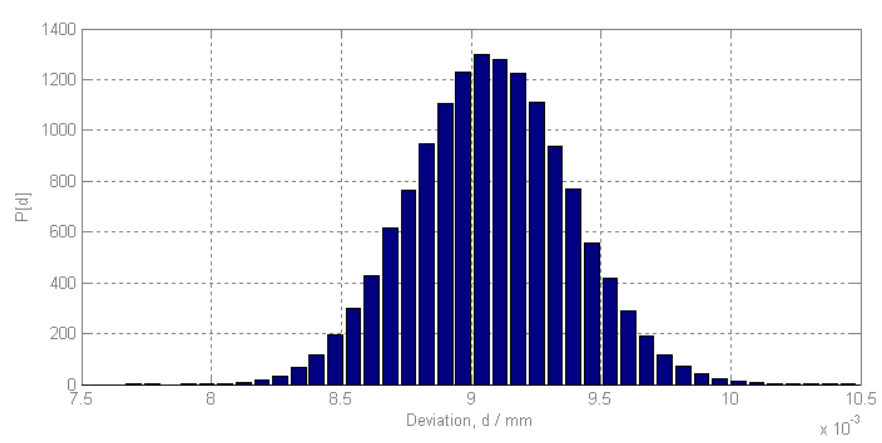

Fig. 9. PDF for the $\angle A B$ orthogonality deviation.

calibration uncertainty $\left(U_{95 \%}=30^{\prime \prime}\right)$ with the measurement repeatability. The results obtained are found in Table 7.

The values obtained, combining the estimate and the uncertainty interval have an order of magnitude less than $1 / 10$ th of the tolerance, allowing to assure that the conformity assessment can be properly done by this way.

Based on the output deviation probability density function (Fig. 9) obtained using MCM it was possible to evaluate if the nature of the mathematical model, including a trigonometric function, would introduce any asymmetry or change of the Gaussian shape. The evaluation of skewness and kurtosis confirm the expected normality of the output distribution.

\section{Conclusions}

Metrology probabilistic approach has mathematical and computational tools particularly suited to improve the quality of measurement thus fulfilling the growing technological demands of the modern society.

In the context of civil engineering, where diverse frameworks can be found, measurement is often a major support for the analysis and decision-making, whether it concerns simply the relative quality of products or processes up to extreme cases where integrity, safety and risk assessment determines the establishment of special requirements for conformity assessment.

Thus, the metrological analysis and, in particular, the evaluation of measurement uncertainty, can have a role in the process of Quality assurance, which should be one of the aims of the activity related to Research, Development and Innovation. 


\section{References}

1. ISO/TS 17892-9:2004 Geotechnical investigation and testing - Laboratory testing of soil - Part 9: Consolidated triaxial compression tests on water saturated soil

2. ISO/TS 28037:2010 The determination and use of straight-line calibration functions, Technical Specification, International Organization for Standardization, Geneva

3. BS EN 12390-1: 2002 Testing hardened concrete - Part 1: Shape, dimensions and other requirements for specimens and moulds
4. EN 196-1: 2005. Methods of testing cement - Part 1: Determination of strength

5. JCGM. Evaluation of measurement data - Guide to the expression of uncertainty in measurement, JCGM 100:2008

6. JCGM. Evaluation of measurement data - Supplement 1 to the "Guide to the expression of uncertainty in measurement" - Propagation of distributions using a Monte Carlo method, JCGM 101: 2008

7. JCGM. Evaluation of measurement data - Supplement 2 to the "Guide to the expression of uncertainty in measurement" - Extension to any number of output quantities, JCGM 102: 2011 